\title{
Response of a C-Shaped Building Under Blast Loadings
}

\author{
Kanchan Pujari, D.H.Tupe, G.R.Gandhe
}

\begin{abstract}
Our structures especially building structures, are designed basically for dead loads, live loads for static loading conditions and for wind, earthquake and their combinations for dynamic lateral loadings. As the structures are not designed for unexpected blast load conditions it is not possible for the structures to resist such destructive forces of blast impacts. In this research work a comparative study has been done for the blast forces and earthquake forces. For this study purpose a $C$-shaped building was modelled for the analysis in finite element program SAP-2000. Initially the building model was analysed for Dead loads, live loads and Earthquake loads. In the second model the building is designed for Blast load. Blast load was defined as a triangular function using codal provisions suggested by IS-4991. The results then are compared and it was checked how far a building designed for $E Q$ can withstand a blast of specific amount of TNT. Also the effect of blast forces due to shape of the building is checked. The comparative study has been mapped and indicated in the paper.
\end{abstract}

Index Terms: Blast Loads, Earthquake loads, Non Linear analysis, SAP-2000, Time History Analysis.

\section{INTRODUCTION}

Structures such as buildings, bridges, water tanks in general are basically designed to resist various forces due to the dead loads, live loads and some lateral forces caused due to wind or seismic activities. In the past few decades due to imbalanced social harmony there has been a lot of terrorist attacks, especially in India. These attacks were in the form of blasts. When the structures are enforced with such blasts they cause serious deformations which may even prove to be fatal for the structures. Such fatal attacks causes serious losses to lives and properties. For this purpose the structures which are expected to undergo such conditions must be designed for such critical fatal loading conditions. To predict such loads is not an easy task and hence, the buildings are not generally designed to withstand such criteria.

The disastrous bombings in U.S. embassies, Nairobi in 1998, the funeral building of Murrah, Oklahoma 1995, and the WTC bombings in 1993 had caused several losses. The charge weight of Tri-nitro-toluene (TNT) was around $800-850 \mathrm{~kg}$. Due to the increase in such activities there has been rise of numerous studies which have been researching solutions to such complicated problems. From the past

Revised Version Manuscript Received on August 19, 2019.

Kanchan Pujari, P.G. Student, Civil Engineering Department, Deogiri Institute of Engineering and Management Studies, Aurangabad, Maharashtra, India.

Prof. D.H.Tupe, Assistant professor, Civil Engineering Department, Deogiri Institute of Engineering and Management Studies, Aurangabad, Maharashtra, India.

Dr. G.R.Gandhe, Professor, Civil Engineering Department, Deogiri Institute of Engineering and Management Studies, Aurangabad, Maharashtra, India. attacks and incidents it was realised that every important buildings such as public buildings, government owned institutions, and other such important buildings must be designed for adequate resistance to such loading conditions.

This study is also related to the effects of blast loads on a structure which has a C-shaped plan. Basically in the seismically activated zones we are ought to design the structures for seismic forces. In this study a simulation between earthquakes induced forces and blast forces has been studied. The capacity of a structure which is designed to resist earthquakes in an activated vulnerable zone, to resist blast loads is checked. Lastly on this basis few conclusions have been drawn that if a structure which is designed for EQ forces can withstand a specific amount of blast loads.

A. Blast Phenomenon, Earthquake Loadings and Indian Standards.

From the past studies it can be rightly said that the earthquake and blast have many differences but some similarities too. Both the earthquake forces and the blast forces are dynamic in nature. Also the structural response to these forces is inelastic as well [1]. On the other side there are some differences in both these loads. The blast loads are acting on the structure above the ground where as EQ forces acts below the ground. The forces due to EQ are cyclic whereas the forces due to blast loads are impulsive [5]. 

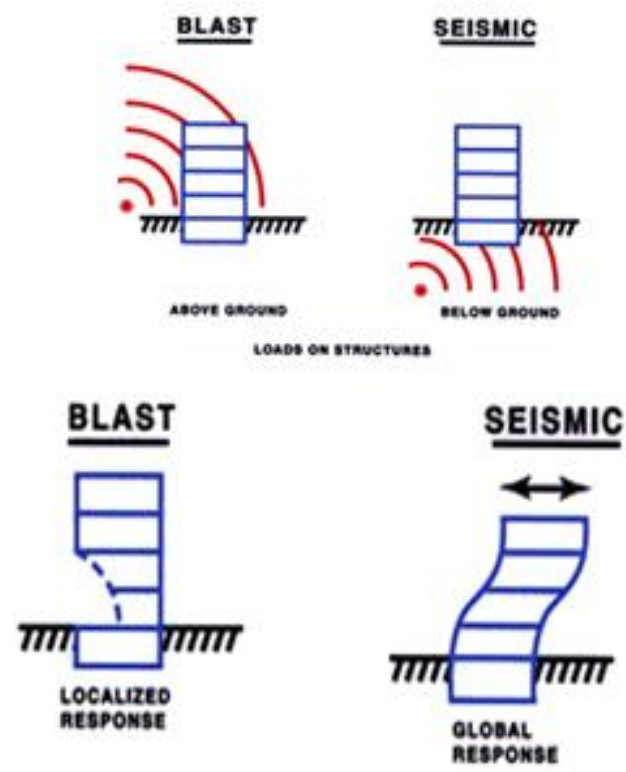

sthuctuRt hesponst

BLAST

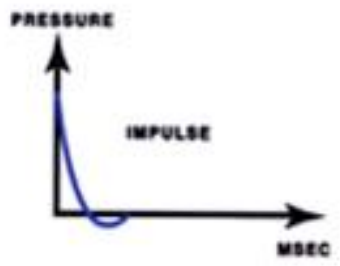

SEISMIC

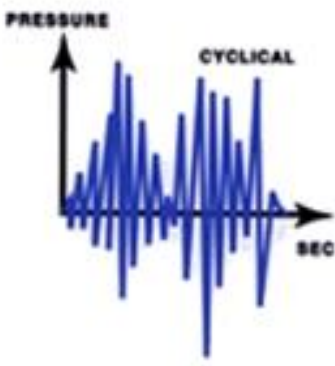

CAD asomatuas

Figure 1: Differences in Blast and EQ (IS-4991)

\section{B. Ideal blast wave characteristics}

An explosion may be stated as a fast chemical reaction involving a solid, dust or gas, during which a rapid release of hot gases and energy takes place. This phenomenon lasts only for some milliseconds and it results in the production of very high pressures and temperatures. During detonation the hot gases that are produced expand in order to occupy the available space, leading to wave type propagation through space that is transmitted spherically through an unbounded surrounding medium. Along with the produced gases, the air around the blast (for air blasts) also expands and its molecules pile-up, resulting in what is known as a blast wave and shock front. The blast wave contains a large part of the energy that was released during detonation and moves faster than the speed of sound.

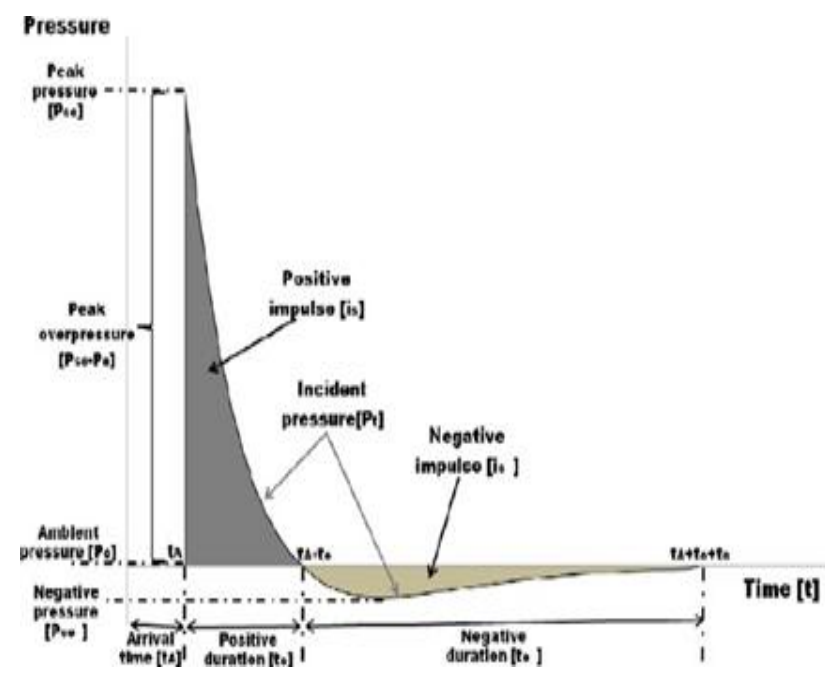

Figure 2: Idealised Curve for Blast Phenomenon

Fig. 2, Shows the phenomenon of the blast loads acting. It can be seen that the idealised profile of a blast is a triangular function of pressure with respect to time. The Pressure surrounding the element is initially equal to the ambient pressure Po, further it undergoes an instantaneous increase to a peak pressure Pso at the arrival time tA, when the shock front reaches that point. The time needed for the pressure to reach its peak value is very small and for design purposes it is assumed to be equal to zero. The peak pressure Pso is also known as side-on overpressure or peak overpressure. The value of the peak overpressure as well as the velocity of propagation of the shock wave decrease with increasing distance from the detonation centre. After its peak value, the pressure decreases with an exponential rate until it reaches the ambient pressure at tA+to, to being called the positive phase duration. After the positive phase of the pressure-time diagram, the pressure becomes smaller (referred to as negative) than the ambient value, and finally returns to it. The negative phase is longer than the positive one, its minimum pressure value is denoted as Pso and its duration as to during this phase the structures are subjected to suction forces, which is the reason why sometimes during blast loading glass fragments from failures of facades are found outside a building instead in its interior. The negative phase of the explosive wave is usually not taken into account for design purposes as it has been verified that the main structural damage is connected to the positive phase. [2]

Additionally, the pressures that are produced from the negative phase of the blast wave are relatively small compared to those of the positive phase and since these are in the opposite direction, it is usually on the safe side to assume that they do not have a big impact on the structural integrity of buildings under blast loads. [2] 


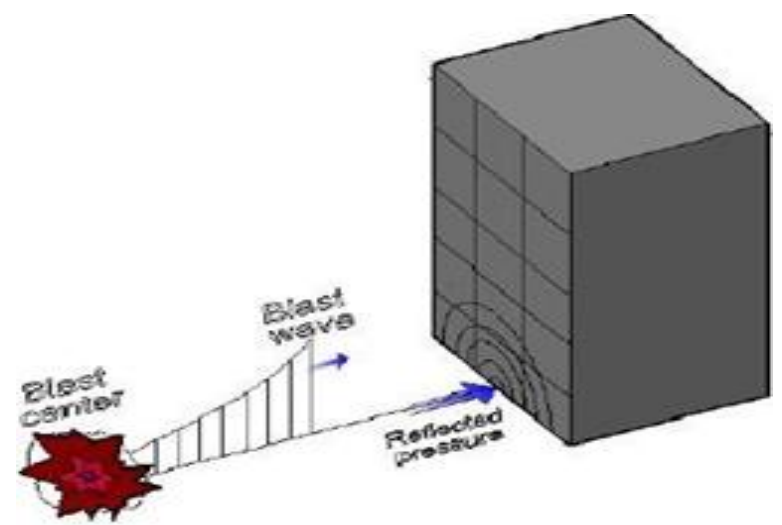

Figure 3: Blast wave acting on the structure

\section{II.SYSTEM DEVELOPMENT}

A G+9 storied, reinforced concrete building was considered for the study. Table $1 \& 2$ shows the material and structural specifications of the structure, used for the study.

Table 1: Material Properties considered

\begin{tabular}{|c|c|c|}
\hline 1. & Grade of concrete & M35 \\
\hline 2. & Grade of reinforcing steel & Fe 500 \\
\hline 3. & Density of concrete & $25 \mathrm{KN} / \mathrm{m}^{3}$ \\
\hline 4. & Density of brick masonry & $19 \mathrm{KN} / \mathrm{m}^{3}$ \\
\hline 5. & Damping ratio & $5 \%$ \\
\hline
\end{tabular}

Table 2: Structural Specifications

\begin{tabular}{|c|l|c|}
\hline 1. & Plan Type & C-Shaped \\
\hline 2. & Plan Dimensions & $(30 \mathrm{X} 21) \mathrm{m}$ \\
\hline 3. & No. of bays along X/Y & $10 / 7$ \\
\hline 4. & Width of each bay & $3.0 \mathrm{~m}$ \\
\hline 5. & Height of the structure & $30 \mathrm{~m}$ \\
\hline 6. & Height of each storey & $3.0 \mathrm{~m}$ \\
\hline 7. & Thickness of Slabs & $150 \mathrm{~mm}$ \\
\hline 8. & Internal / External Wall thickness & $150 \mathrm{~mm}$ \\
\hline 9. & Depth of footings & $3 \mathrm{~m}$ \\
\hline 10. & Zone (IS-1893) & IV \\
\hline 11. & Importance factor & 1.0 \\
\hline 12. & Response reduction factor & 5.0 \\
\hline 13. & Site soil type & Medium (II) \\
\hline
\end{tabular}

\subsection{METHODOLOGY}

For the study purpose a $100 \mathrm{~kg}(0.1 \mathrm{~T})$ TNT was considered for the blast with a stand-off distance of $30 \mathrm{~m}$. As per the various literatures studied the blast loads are most suitably indicated using Time History loadings [3]. The peak pressure values were calculated according to IS-4991 (1968). The pressures then were applied on the structural elements such as columns and beams which were facing towards the blast on the front and either sides of the building as shown in fig.5.

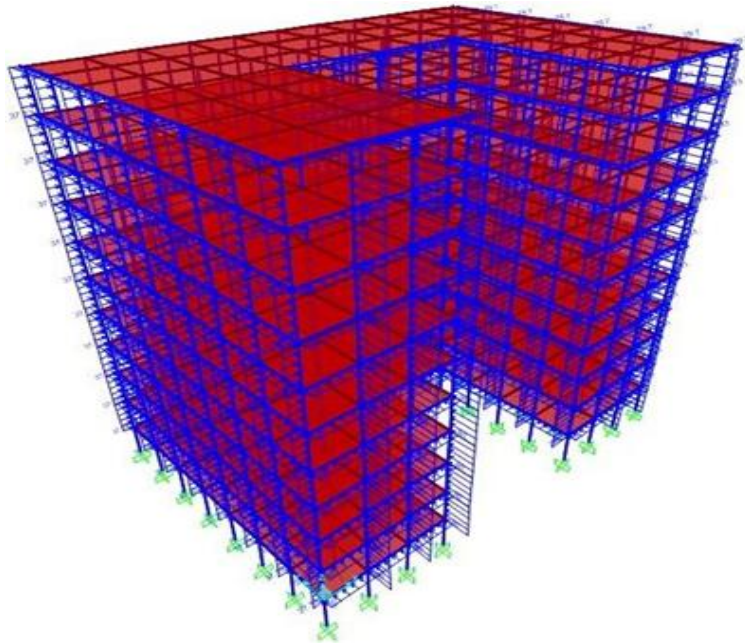

Figure 6: Blast loads as UDL on Structural Elements

\subsection{BLAST LOADS AS PER IS-4991}

For $100 \mathrm{~kg}$ TNT at $30 \mathrm{~m}$ stand-off distance, the various parameters have been considered as below:

- Scaled Distance $(\mathrm{X})=64.63 \mathrm{~m}$

- Peak Side Overpressure (Pso) $=0.353 \mathrm{~kg} / \mathrm{cm} 2$

- Mach No. $(\mathrm{M})=1.144$

- Positive Phase Duration (T0) $=17.52$ Mil. Sec.

- Eq. Trian.Pulse Duration $(\mathrm{Td})=13.16 \mathrm{Mil}$. Sec.

- Dynamic Pressure ratio T0/Td = 0.041

- Peak Reflected Overpressure Ratio = 0.792

All the above calculations have been done according to

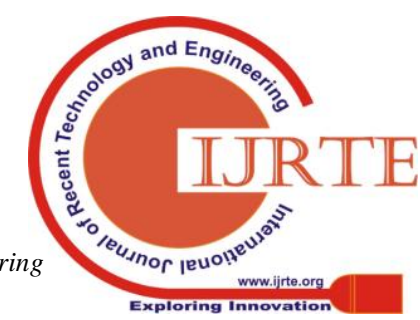


table no.1, IS-4991. All these pressures are calculated for idealised

positive phase only. No negative phase pressure is considered as it shows relatively lesser pressure values [2]. Non Linear Analysis has been performed using Finite element software SAP-2000. The Number of steps was considered to be 3500 and each step size was 0.01 for the sake of better improved accuracy

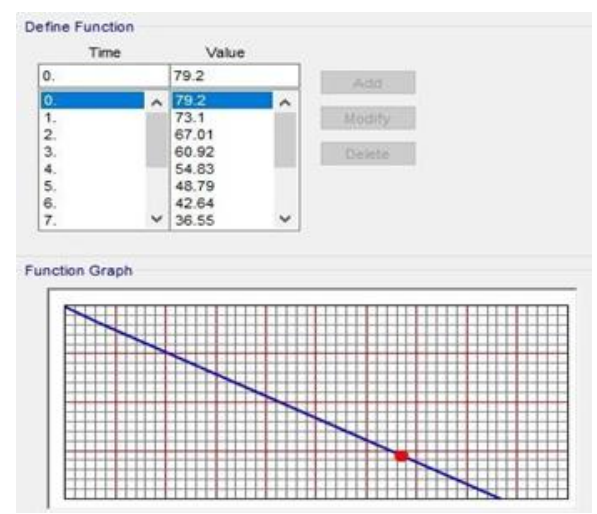

Figure 7: Blast Pressure at front face

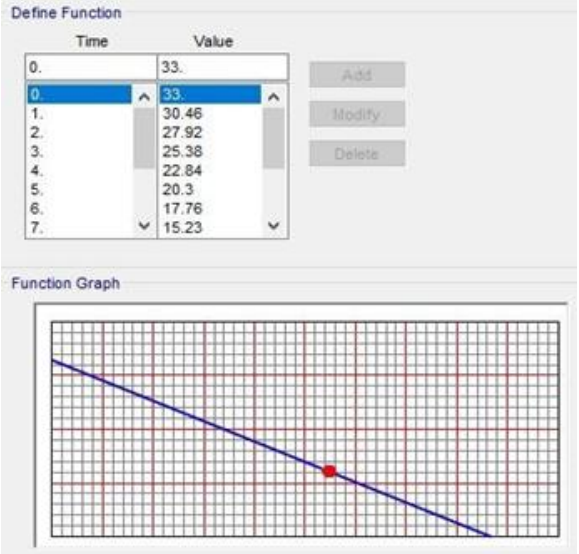

Figure 5: Blast Pressure at Side faces

\section{RESULTS \& DISCUSSION.}

\subsection{Displacement Comparison}

The displacements of the structure were checked for all the three models. The first models displacement was carried out for only earthquake loadings. The second model was designed to earthquake resistant and blast load was applied and its displacements were checked. In the third model the structure was infilled with shear walls on the exterior faces and displacement results for it was checked. Fig. 8 shows the comparison of displacements of various stories with respect to the type of loadings applied on the specified structures.

It can be seen from the fig.8, that the displacements of the stories for the structure with only earthquake loads is very less. Secondly it is observed that when a structure is applied with blast load caused due to $100 \mathrm{~kg}$ TNT at $30 \mathrm{~m}$ stand-off distance, the structure shows much greater displacements. Lastly the structures were coupled with a shear wall of 230 mm thickness and M35 grade of concrete. It was seen that due to the application of shear walls in the structures around eighty six percent displacements were reduced.

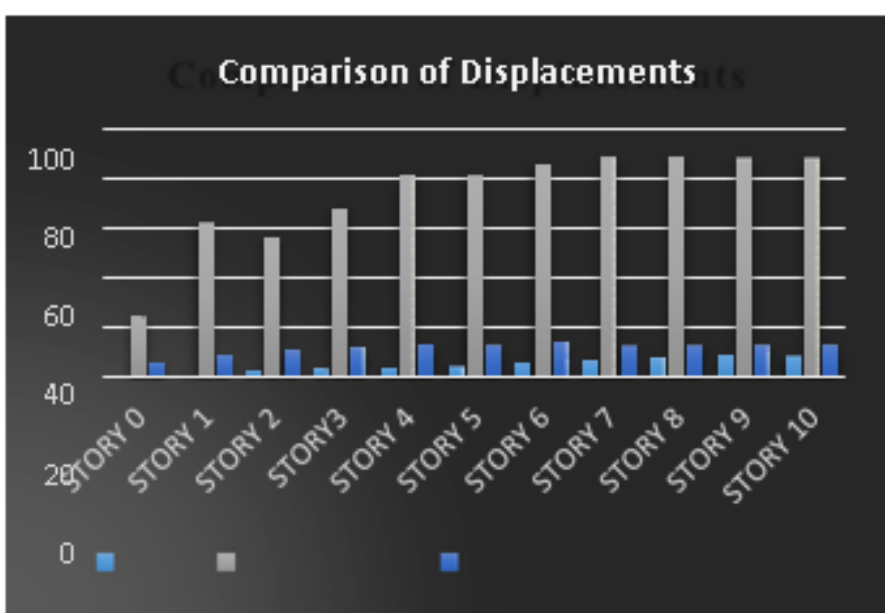

Figure 8: Comparison of Displacements

\subsection{Acceleration vs Time functions}

After the displacements were check the next result was checked for the story accelerations. The figures shown below indicates results for the acceleration of various models i.e. fig.9 indicates acceleration for the structure with only earthquake forces. Fig.10, 11, indicates the accelerations regarding the blast load models and structure coupled with shear walls respectively.

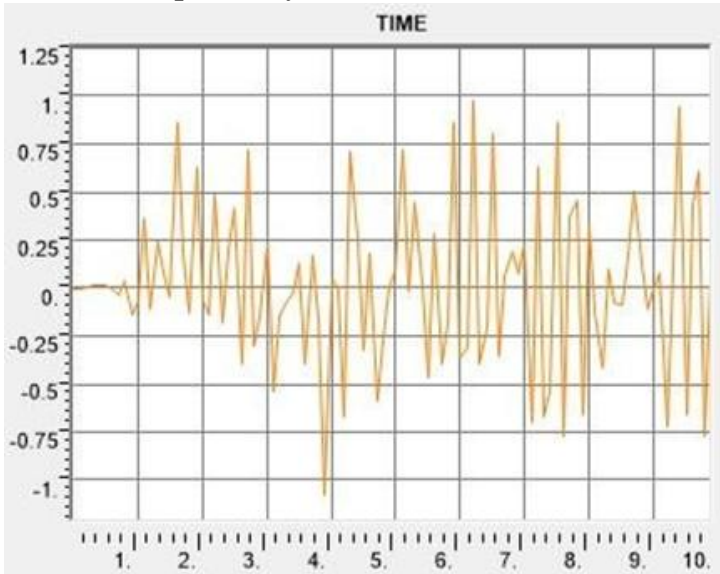

Figure 9: Acceleration for EQ loadings

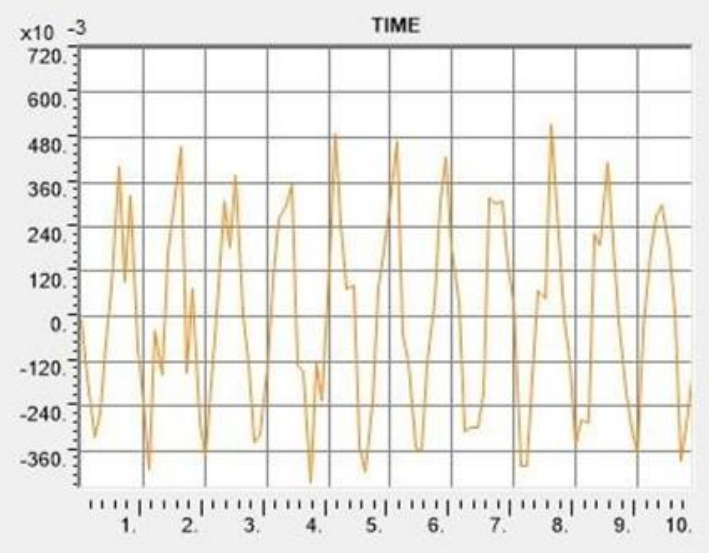

Figure 10: Acceleration for Blast Load 


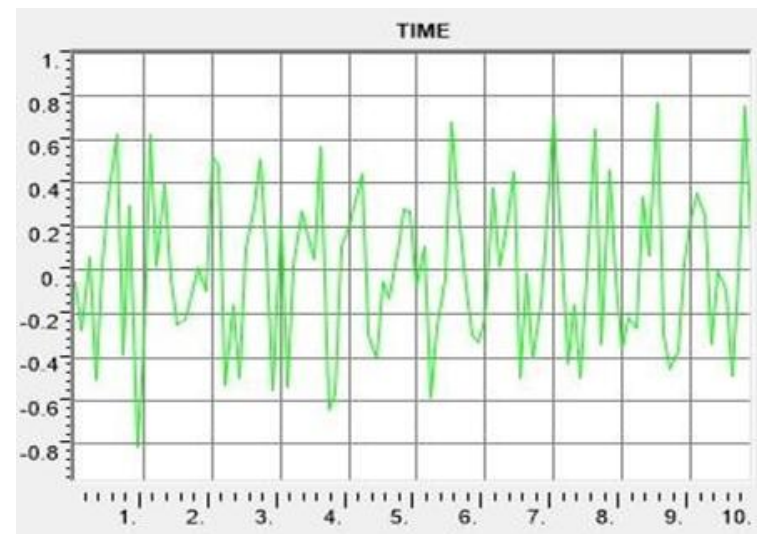

Figure 11: Acceleration for structure with shear walls and blast loads

It was observed from the acceleration vs time functions of all the three models that the accelerations due to blast loads are even more destructive than that of the earthquake loading conditions. It was also seen that due to the application of shear walls in the structure the accelerations reduced to a considerable extent making the structure comparably safer for the blast loads.

\section{CONCLUSIONS}

- The Response of RC structures to blast loads and EQ loads is quite different.

- The displacement variation is non-uniform along the vertical axis of the building.

- If a structure is designed for earthquake resistance and blast are applied the structure performs well.

- The structure if coupled with shear walls on the periphery of the building and then blast loads are applied the structure performs very well.

- The displacements for structure with shear walls applied with blast loads reduced up to $87 \%$.

- It can be suggested that if a building is designed for EQ and shear walls are applied, it can perform well for $100 \mathrm{~kg}$ TNT blast with a stand-off distance of $30 \mathrm{~m}$

\section{REFERENCES}

1. Aditya C. Bhatt et al., "Comparative Study of Response of Structures Subjected To Blast and Earthquake Loading", Int. Journal of Engineering Research and Applications, Vol. 6, Issue 5, May 2016, pp.62-66.

2. JRC 32253-2011, Blast Simulation Technology Development, European Commission Joint Research Centre Institute for the Protection and Security of the Citizen, 201.

3. Zubair I. Syed, "Performance of Earthquake-resistant RCC Frame Structures under Blast Explosions", Procedia Engineering 180, Science direct, May, 2016, pp. 82-90

4. Quazi Kashif, "Effect of Blast on G+4 RCC Frame Structure", International Journal of Emerging Technology and Advanced Engineering, Volume 4, Issue 11, November 2014, pp-145-149

5. IS Code IS: 4991-1968 - Criteria for blast resistant design of structures for explosion above groundll, Bureau of Indian Standards (1968) 